Research Article

\title{
SPECIES COMPOSITION, DISTRIBUTION, LIFE FORMS AND FOLK NOMENCLATURE OF FOREST AND COMMON LAND PLANTS OF WESTERN CHITWAN, NEPAL
}

\author{
D. R. Dangol \\ Institute of Agriculture and Animal Sciences, Rampur, Chitwan, Nepal \\ Email: dharmadangol@hotmail.com
}

\begin{abstract}
This paper enumerates 349 plant species belonging to 77 families of vascular plants collected in the winter seasons of 1996 and 2000 by the flora teams of the Population and Ecology Research Laboratory, Nepal. Of the total species, 249 species belong to dicotyledons, 87 species to monocotyledons and 13 species to pteridophytes. Among the families, dicotyledons contributed the highest number of families (55 in number) followed by monocotyledons and pteridophytes. In the study areas, species composition varies with the type of habitats in the study plots. Some species are unique in distribution. The highest unique species are contributed by common lands (87 spp.), followed by the Chitwan National Park forest (36 spp.) and Tikauli forest (32 spp.). Ageratum houstonianum Mill., Cynodon dactylon (L.) Pers., Imperata cylindrica (L.) Beauv., Rungia parviflora (Retz.) Nees, Saccharum spontaneum L. and Thelypteris auriculata (J. Sm.) K. Iwats are the most common species across all the research blocks. Of the listed plants, many plants have local names either in Nepalese or other tribal languages. Plants are named in different ways on the basis of habit, habitat, smell, taste, and morphological characters of the plants, which are also the basis of nomenclature in plant taxonomy.
\end{abstract}

Key words: Folk name, plant biodiversity, plant distribution, species composition analysis

\section{INTRODUCTION}

Plants, people and culture are very closely linked with each other and one factor affects others in any ecosystem of the world (Bhuju and Rana, 2000; Cosgriff et al., 1999; Dinerstein, 1992, Ghimire et al., 1999; Roy and Pokharel, 2000). The dependence of people on the plants of any area is affected by many biotic and environmental factors. The utilization of plants also depends on the knowledge of the people, and their choice of resources for their existence (IUCN, 1994). Therefore, people of any area are the key factors for the loss or replacement or introduction of the plant diversity from/in a particular place. To understand what, how, and when people extract/exploit the plant wealth for the benefit of the people or understanding the status of the plants and people relationship, first we have to know the kinds of flora and vegetation of a particular locality (Penitsa et al., 1994; Turner, 1997). We also have to explore how the people and environment are interacting with each other. In this context we have collected data on population and the environment to understand the reciprocal relation of people, plants and the environment at micro-level. Matthews et al. (2000) examined reciprocal relation of population and the environment in the western Chitwan. In this paper, plant specimens collected in winter seasons of 1996 and 2000 will be dealt with to highlight species diversity, life forms, distribution and folk nomenclature of plants in the selected study areas of western Chitwan.

\section{METHODOLOGY}

Plant specimens were collected from research plots located in three forests and 138 common lands of western Chitwan (Map 1). The forest plots were distributed in three natural forests and three Dalbergia sissoo plantation forests. Of the three natural forests, one forest lies in the Royal Chitwan National Park, the first national park of Nepal which is also cited in CITES list. Ten other plots were located in the savanna grassland of the same national park. The common land plots were scattered in 38 neighborhoods. The present list is prepared from the plants encountered inside the research plots of different sizes $\left(10 \times 10 ; 3 \times 3\right.$; and $\left.1 \times 1 \mathrm{~m}^{2}\right)$. This catalogue includes all the plant resources of the forests and common lands of western Chitwan listed in the flora forms and the specimens collected by flora teams supervisors and author himself during JanuaryApril, 1996 and January-April, 2000. 
Nomenclature follows the "Annotated checklist of the flowering plants of Nepal" (Press et al., 2000), "Enumeration of the vascular plants of west Nepal" (Rajbhandari et al., 1994), "The plant book: a portable dictionary of the higher plants" (Mabberley, 1987) and "CRC world dictionary of plant names" (Quattrochhi, 2000).

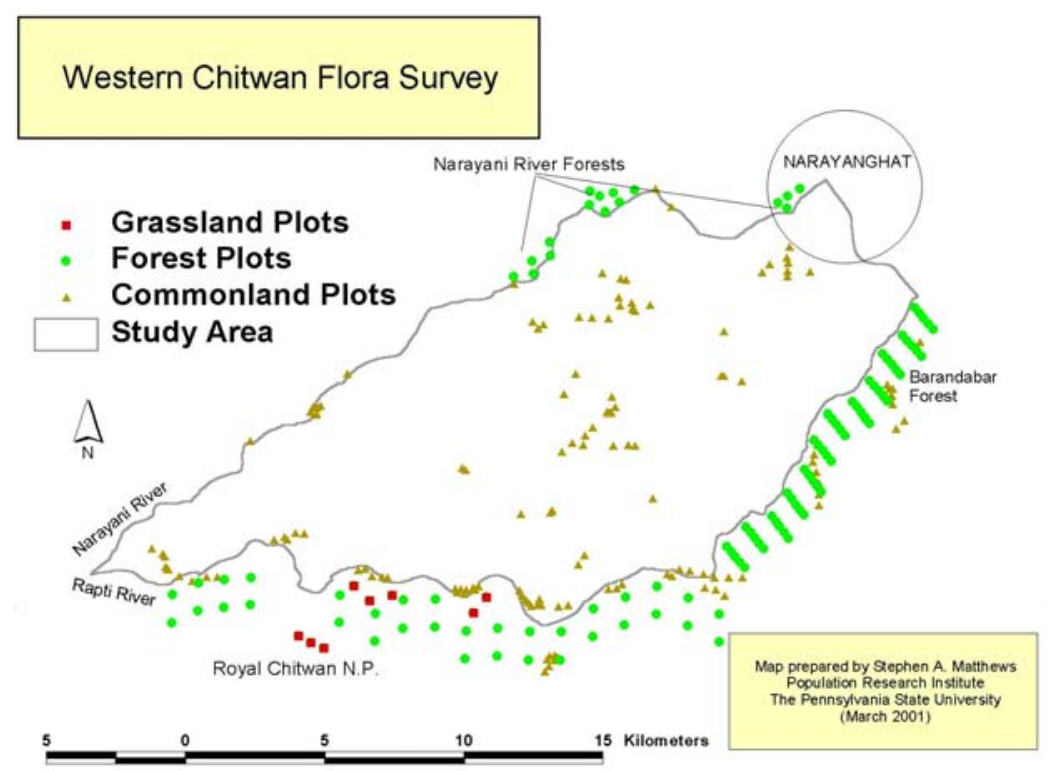

Map 1. Flora plots in western Chitwan, Nepal

\section{RESULTS AND DISCUSSION}

\section{Species composition analysis}

This checklist includes a total of 349 species belonging to 249 genera and 77 families of vascular plants of western Chitwan. Among the taxa, dicotyledons contributed the highest number of families (55), genera (180) and species (249), followed by monocotyledons and pteridophytes (Figure 1). Earlier studies also reported the Poaceae as the most important family in Rampur condition (Dangol, 1998-1999; Dangol and Gurung, 1998: 1989). Asteraceae was reported as the largest family in the flora of Nepal (Hara et al. 1978, 1979, 1982) and Leguminosae recorded as the largest family in Morang district and adjoining areas of Nepal (Jha and Jha, 2000). This indicates that the dominance of a family varies from place to place. The Poaceae are the largest family in terms of number of genera and species, followed by Leguminosae, Asteraceae, Cyperaceae, Labiatae, Rubiaceae, Scrophulariaceae and Euphorbiaceae (Table 1). The Acanthaceae, Commelinaceae, Tiliaceae, Verbenaceae and Boraginaceae also increase the diversity of species in the study areas. The families with few species are comparatively more, for example, 6 families of pteridophytes, 20 of dicotyledons and 5 of monocotyledons contributed one species each (Table 2).

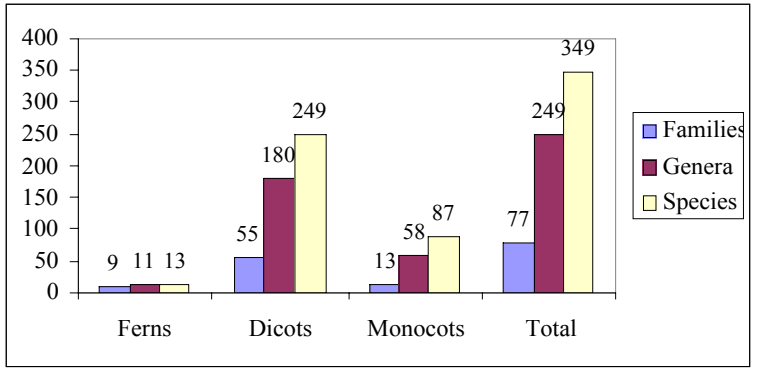

Figure 1. Floristic analysis of plants of western Chitwan 
Table 1. Families with the largest number of genera and species in the vascular flora of western Chitwan, Nepal

\begin{tabular}{|lcc|}
\hline Families & Genera & Species \\
\hline Poaceae & 31 & 45 \\
Leguminosae & 23 & 39 \\
Asteraceae & 29 & 36 \\
Cyperaceae & 9 & 16 \\
Rubiaceae & 7 & 15 \\
Scrophulariaceae & 7 & 15 \\
Labiatae & 10 & 15 \\
Euphorbiaceae & 6 & 10 \\
Acanthaceae & 7 & 9 \\
Commelinaceae & 4 & 8 \\
\hline
\end{tabular}

Table 2. Species checklist of the plants of western Chitwan, Nepal

\begin{tabular}{|c|c|c|c|c|}
\hline Family & Specific epithet & Nepali name & Life form & Distribution \\
\hline Acanthaceae & Barleria cristata L. & Kuro & $\mathrm{S}$ & TF, RF, CL \\
\hline Acanthaceae & Hemigraphis birta (Vahl) T. Anderson & & $\mathrm{H}$ & $\mathrm{TF}, \mathrm{RF}$ \\
\hline Acanthaceae & Hygrophila auriculata (Schumach.) Hein & & $\mathrm{H}$ & CL \\
\hline Acanthaceae & Hygrophila polysperma (Roxb.) T. Anderson & & $\mathrm{H}$ & $\mathrm{CL}$ \\
\hline Acanthaceae & Justicia quinqueangularis Koenig. & & $\mathrm{H}$ & CL \\
\hline Acanthaceae & Justicia sp. & & $\mathrm{H}$ & $\mathrm{NF}$ \\
\hline Acanthaceae & Lepidagathis incurva Buch.-Ham. ex D. Don & Bankuro & $\mathrm{H}$ & $\mathrm{NF}$ \\
\hline Acanthaceae & Nelsonia canescens (Lam.) Spreng. & & $\mathrm{H}$ & $\mathrm{RF}$ \\
\hline Acanthaceae & Rungia parviflora (Retz.) Nees & Runche jhar & $\mathrm{H}$ & TF, RF, NF, GL, CL \\
\hline Amaranthaceae & Achyranthes aspera $\mathrm{L}$. & Datiwan & $\mathrm{H}$ & TF, NF, CL \\
\hline Amaranthaceae & Aerva lanata Juss. & & $\mathrm{H}$ & $\mathrm{NF}$ \\
\hline Amaranthaceae & Alternanthera sessilis (L.) DC. & Bhiringi jhar & $\mathrm{H}$ & CL \\
\hline Anacardiaceae & Buchanania latifolia Roxb. & Bhalayo & $\mathrm{T}$ & $\mathrm{TF}$ \\
\hline Anacardiaceae & Semecarpus anacardium L. f. & Bhalayo & $\mathrm{T}$ & $\mathrm{TF}$ \\
\hline Apiaceae & Centella asiatica (L.) Urb. & Ghodtapre & $\mathrm{H}$ & TF, NF, GL, CL \\
\hline Apiaceae & Hydrocotyle sibthorpioides Lam. & Sano ghodtapre & $\mathrm{H}$ & $\mathrm{RF}, \mathrm{NF}, \mathrm{CL}$ \\
\hline Apiaceae & Oenanthe javanica (Blume) DC. & & $\mathrm{H}$ & $\mathrm{RF}, \mathrm{CL}$ \\
\hline Apocynaceae & Alstonia scholaris (L.) R. Br. & Chhatiwan & $\mathrm{T}$ & CL \\
\hline Apocynaceae & Holarrhena pubescens (Buch.-Ham.) Wall. ex G. Don & Dudhkhirro & $\mathrm{T}$ & $\mathrm{TF}, \mathrm{RF}$ \\
\hline Apocynaceae & Trachelospermum lucidum (D. Don) K. Schum. & Salikal & CS & $\mathrm{RF}$ \\
\hline Apocynaceae & Vallaris solanacea (Roth) Kuntze & Dudhe lahara & CS & $\mathrm{TF}, \mathrm{NF}$ \\
\hline Araceae & Colocasia esculenta (L.) Schott. & Pidalu & $\mathrm{H}$ & $\mathrm{RF}$ \\
\hline \multirow[t]{2}{*}{ Arecaceae } & Phoenix humilis Royle ex Becc. \& Hook. f. & Dhotipate/ & & \\
\hline & & Thakal & DT & TF, RF \\
\hline Asclepiadaceae & Calotropis gigantea (L.) Dryand & Aank & $\mathrm{S}$ & $\mathrm{CL}$ \\
\hline Asteraceae & Adenostemma lavenia (L.) Kuntze & & $\mathrm{H}$ & RF, CL. \\
\hline Asteraceae & Ageratum conyzoides L. & Gandhe (Seto) & $\mathrm{H}$ & $\mathrm{NF}, \mathrm{CL}$ \\
\hline Asteraceae & Ageratum houstonianum Mill. & Gandhe (Nilo) & $\mathrm{H}$ & TF, RF, NF, GL, CL \\
\hline Asteraceae & Artemisia dubia Wall. ex Besser & Titepati & $\mathrm{H}$ & $\mathrm{NF}$ \\
\hline Asteraceae & Bidens pilosa L. & Kalo kuro & $\mathrm{H}$ & CL \\
\hline Asteraceae & Blumea lacera (Burm. f.) DC. & Thulo mulapate & $\mathrm{H}$ & $\mathrm{CL}$ \\
\hline Asteraceae & Blumea laciniata DC. & Thulo mulapate & $\mathrm{H}$ & TF, RF, GL, CL \\
\hline Asteraceae & Blumea sp. & Thulo mulapate & $\mathrm{H}$ & $\mathrm{RF}$ \\
\hline Asteraceae & Blumeopsis flava (DC.) Gagnep. & Torigande & $\mathrm{H}$ & TF, RF, CL \\
\hline Asteraceae & Breea arvensis (L.) Less. & Gainda kande & $\mathrm{H}$ & TF, RF, CL \\
\hline Asteraceae & Caesulia axillaris Roxb. & Thuk jhar & $\mathrm{H}$ & $\mathrm{CL}$ \\
\hline Asteraceae & Centipeda minima (L.) A. Braun \& Asch. & & $\mathrm{H}$ & CL \\
\hline
\end{tabular}


Table 2. Cont.

\begin{tabular}{|c|c|c|c|c|}
\hline Family & Specific epithet & Nepali name & Life form & Distribution \\
\hline Asteraceae & Conyza canadensis (L.) Cronquist & Mulapate & $\mathrm{H}$ & NF, CL \\
\hline Asteraceae & Conyza leucantha (D. Don) Ludlow \& R. H. Raven & Mulapate & $\mathrm{H}$ & $\mathrm{NF}$ \\
\hline Asteraceae & Eclipta prostrata (L.) L. & Bhringraj & $\mathrm{H}$ & TF, RF, GL, CL \\
\hline Asteraceae & Elephantopus scaber L. & Thinko & $\mathrm{H}$ & TF, RF \\
\hline Asteraceae & Emilia sonchifolia (L.) DC. & Mulapate & $\mathrm{H}$ & CL \\
\hline Asteraceae & Eupatorium odoratum $\mathrm{L}$. & Banmara & $\mathrm{H}$ & TF, NF \\
\hline Asteraceae & Galinsoga quadriradiata Ruiz \& Pav. & Chitlange jhar & $\mathrm{H}$ & $\mathrm{CL}$ \\
\hline Asteraceae & Gnaphalium affine D. Don & Boki jhar & $\mathrm{H}$ & $\mathrm{CL}$ \\
\hline Asteraceae & Gnaphalium polycaulon Pers. & Boki jhar & $\mathrm{H}$ & $\mathrm{RF}, \mathrm{CL}$ \\
\hline Asteraceae & Gnaphalium purpureum $\mathrm{L}$. & Boki jhar & $\mathrm{H}$ & $\mathrm{CL}$ \\
\hline Asteraceae & Grangea maderaspatana (L.) Poir. & & $\mathrm{H}$ & CL \\
\hline Asteraceae & Hemistepta lyrata Bunge & & $\mathrm{H}$ & $\mathrm{NF}$ \\
\hline Asteraceae & Inula rubricaulis (DC.) C. B. Clarke & Kan pate & $\mathrm{H}$ & TF, RF \\
\hline Asteraceae & Ixeris polycephala Cass. & Dudhe jhar & $\mathrm{H}$ & NF, CL \\
\hline Asteraceae & Launaea aspleniifolia (Willd.) Hook. f. & Sano mulapate & $\mathrm{H}$ & TF, RF, GL, CL \\
\hline Asteraceae & Launaea procumbens (Roxb.) Ramayya \& Rajagopala & & $\mathrm{H}$ & $\mathrm{CL}$ \\
\hline Asteraceae & Senecio ramosus Wall. ex DC. & & $\mathrm{H}$ & CL \\
\hline Asteraceae & Sphaeranthus indicus L. & & $\mathrm{H}$ & GL, CL \\
\hline Asteraceae & Spilanthes uliginosa Swarts & Marethi & $\mathrm{H}$ & CL \\
\hline Asteraceae & Synedrella nodiflora Gaertn. & Pahele jhar & $\mathrm{H}$ & $\mathrm{NF}$ \\
\hline Asteraceae & Tridax procumbens $\mathrm{L}$. & Putali jhar & $\mathrm{H}$ & CL \\
\hline Asteraceae & Vernonia cinerea (L.) Less. & Jhurjhure & $\mathrm{H}$ & TF, RF, NF, CL \\
\hline Asteraceae & Xantbium strumarium $\mathrm{L}$. & Bhende kuro & $\mathrm{H}$ & TF, CL \\
\hline Asteraceae & Youngia japonica (L.) DC. & Dudhe jhar & $\mathrm{H}$ & $\mathrm{RF}$ \\
\hline Azollaceae & Azolla sp. & Leu & $\mathrm{H}$ & $\mathrm{CL}$ \\
\hline Bombacaceae & Bombax ceiba $\mathrm{L}$. & Simal & $\mathrm{T}$ & RF, NF, GL, CL \\
\hline Boraginaceae & Bothriospermum zeylanicum (J. Jacq.) Druce & & $\mathrm{H}$ & $\mathrm{CL}$ \\
\hline Boraginaceae & Cynoglossum sp. & & $\mathrm{H}$ & GL \\
\hline Boraginaceae & Heliotropium strigosum Willd. & Hanthi sunde & $\mathrm{H}$ & CL \\
\hline Boraginaceae & Trichodesma indicum (L.) R. Br. & Gerguj & $\mathrm{H}$ & GL \\
\hline Boraginaceae & Ehretia laevis Roxb. & Dhatrung & $\mathrm{T}$ & TF, RF, NF \\
\hline Burseraceae & Garuga pinnata Roxb. & Dabdabe & $\mathrm{T}$ & $\mathrm{TF}, \mathrm{RF}$ \\
\hline Campanulaceae & Campanula pallida Wall. & Ghante jhar & $\mathrm{H}$ & $\mathrm{CL}$ \\
\hline Campanulaceae & Lobelia alsinoides Lam. & & $\mathrm{H}$ & TF, CL \\
\hline Campanulaceae & Wablenbergia marginata (Thunb.) A. DC. & Nilo tike & $\mathrm{H}$ & $\mathrm{CL}$ \\
\hline Cannabaceae & Cannabis sativa $\mathrm{L}$. & Ganja & $\mathrm{H}$ & NF, CL \\
\hline Caryophyllaceae & Polycarpon prostratum (Forssk.) Asch. \& Schweinf. ex Asch. & & $\mathrm{H}$ & $\mathrm{CL}$ \\
\hline Celastraceae & Maytenus thomsonii (Kurz.) Raju \& Babu & Deri & $\mathrm{H}$ & $\mathrm{RF}$ \\
\hline Chenopodiaceae & Chenopodium album $\mathrm{L}$. & Bethe & $\mathrm{H}$ & $\mathrm{CL}$ \\
\hline Combretaceae & Terminalia alata Heyne ex Roth & Saj & $\mathrm{T}$ & $\mathrm{TF}, \mathrm{RF}$ \\
\hline Combretaceae & Terminalia bellirica (Gaertn.) Roxb. & Barro & $\mathrm{T}$ & TF, RF \\
\hline Combretaceae & Terminalia chebula Retz. & Harro & $\mathrm{T}$ & $\mathrm{RF}$ \\
\hline Commelinaceae & Amischophacelus axillaris (L.) Rao \& Kammathy & & $\mathrm{H}$ & $\mathrm{CL}$ \\
\hline \multirow[t]{2}{*}{ Commelinaceae } & Commelina benghalensis $\mathrm{L}$. & Bankane & & \\
\hline & & Jangali kane & $\mathrm{H}$ & TF, RF, NF, CL \\
\hline Commelinaceae & Commelina diffusa Burm. f. & Kane & $\mathrm{H}$ & $\mathrm{TF}$ \\
\hline Commelinaceae & Commelina palludosa Blume & Kane & $\mathrm{H}$ & $\mathrm{TF}$ \\
\hline Commelinaceae & Commelina sp. & Jangali kane & $\mathrm{H}$ & $\mathrm{CL}$ \\
\hline Commelinaceae & Floscopa scandens Lour. & Simkane & $\mathrm{H}$ & CL \\
\hline Commelinaceae & Murdannia nudiflora (L.) Brenan & Kane & $\mathrm{H}$ & $\mathrm{TF}$ \\
\hline
\end{tabular}


Table 2. Cont.

\begin{tabular}{|c|c|c|c|c|}
\hline Family & Specific epithet & Nepali name & Life form & Distribution \\
\hline Commelinaceae & Murdannia spirata (L.) Brueckn. & Kane & $\mathrm{H}$ & TF, CL \\
\hline Convolvulaceae & Argyreia atropurpurea (Wall.) Raizada & & $\mathrm{HC}$ & $\mathrm{TF}$ \\
\hline Convolvulaceae & Evolvulus nummularius (L.) L. & Badampate jhar & $\mathrm{H}$ & TF, CL \\
\hline Convolvulaceae & Merremia kingii Prain & Dudhe jhar & $\mathrm{HC}$ & $\mathrm{TF}$ \\
\hline Convolvulaceae & Merremia vitifolia (Burm. f.) Hallier f. & & HT & $\mathrm{NF}$ \\
\hline Cornaceae & Swida oblonga (Wall.) Sojak & Latikath & $\mathrm{T}$ & TF, RF \\
\hline Cucurbitaceae & Momordica charantia $\mathrm{L}$. & Ban kareli & $\mathrm{HC}$ & $\mathrm{NF}$ \\
\hline Cucurbitaceae & Solena beterophylla Lour. & Gol kankari & $\mathrm{HC}$ & TF, RF \\
\hline Cyperaceae & Carex inanis C. B. Clarke & Mothe & $\mathrm{H}$ & $\mathrm{CL}$ \\
\hline Cyperaceae & Cyperus compressus L. & Mothe & $\mathrm{H}$ & $\mathrm{TF}$ \\
\hline Cyperaceae & Cyperus haspan $\mathrm{L}$. & Mothe & $\mathrm{H}$ & CL \\
\hline Cyperaceae & Cyperus iria L. & Chhate mothe & $\mathrm{H}$ & CL \\
\hline Cyperaceae & Cyperus rotundus L. & Mothe & $\mathrm{H}$ & CL \\
\hline Cyperaceae & Cyperus sp. & Mothe & $\mathrm{H}$ & TF, RF, NF, CL \\
\hline Cyperaceae & Eleocharis pellucida Presl & Jwane jhar & $\mathrm{H}$ & CL \\
\hline Cyperaceae & Fimbristylis dichotoma (L.) Vahl & Mothe & $\mathrm{H}$ & CL \\
\hline Cyperaceae & Fimbristylis falcata (Vahl) Kunth & & $\mathrm{H}$ & $\mathrm{CL}$ \\
\hline Cyperaceae & Kyllinga brevifolia Rottb. & Dalle mothe & $\mathrm{H}$ & CL \\
\hline Cyperaceae & Mariscus aristatus (Rottb.) T. Tang \& F. T. Wang & Sano mothe & $\mathrm{H}$ & $\mathrm{TF}$ \\
\hline Cyperaceae & Mariscus sumatrensis (Retz.) T. Koyama & & $\mathrm{H}$ & $\mathrm{TF}, \mathrm{RF}$ \\
\hline Cyperaceae & Pycreus flavidus (Retz.) T. Koyama & Chiure mothe & $\mathrm{H}$ & CL \\
\hline Cyperaceae & Schoenoplectus juncoides (Roxb.) Palla & Suire jhar & $\mathrm{H}$ & $\mathrm{CL}$ \\
\hline Cyperaceae & Scleria alta Boeck & & $\mathrm{H}$ & $\mathrm{TF}$ \\
\hline Cyperaceae & Scleria parvula Steud. & & $\mathrm{H}$ & $\mathrm{TF}$ \\
\hline Dilleniaceae & Dillenia pentagyna Roxb. & Tantari & $\mathrm{T}$ & TF, RF \\
\hline Dioscoreaceae & Dioscorea bulbifera L. & Ban tarul & $\mathrm{CH}$ & TF, RF \\
\hline Dioscoreaceae & Dioscorea deltoidea Wall. ex Griseb. & Bantarul & $\mathrm{CH}$ & TF, RF \\
\hline Dioscoreaceae & Dioscorea bamiltonii Hook. f. & Bantarul & $\mathrm{CH}$ & $\mathrm{RF}$ \\
\hline Dipterocarpaceae & Shorea robusta Gaertn. & Sal & $\mathrm{T}$ & TF, RF \\
\hline Dryoptericaceae & Diplazium esculentum (Retz.) Sw. ex Shrad. & Neuro & $\mathrm{H}$ & $\mathrm{RF}$ \\
\hline Dryoptericaceae & Tectaria macrodonta (Fee) C. Chr. & Kali neuro & $\mathrm{H}$ & $\mathrm{RF}$ \\
\hline \multirow[t]{2}{*}{ Equisetaceae } & Equisetum debile Roxb. ex Vaucher & Hadjorni/ & & \\
\hline & & Ankhle jhar & $\mathrm{H}$ & RF, NF, GL, CL \\
\hline Eriocaulaceae & Eriocaulon cinereum $\mathrm{R}$. Br. & Buche jhar & $\mathrm{H}$ & $\mathrm{TF}$ \\
\hline \multirow[t]{2}{*}{ Euphorbiaceae } & Aporusa octandra (Buch.-Ham. ex D. Don) & & & \\
\hline & A. R. Vickery ex M. J. Short \& A. R. Vickery & Archal & $\mathrm{S} / \mathrm{T}$ & TF, RF \\
\hline \multirow[t]{2}{*}{ Euphorbiaceae } & Bridelia retusa (L.) Spreng. & Gayo/ & & \\
\hline & & Lahare gayo & $\mathrm{T}$ & $\mathrm{RF}$ \\
\hline Euphorbiaceae & Euphorbia hirta L. & Dudhe jhar & $\mathrm{H}$ & TF, CL \\
\hline Euphorbiaceae & Euphorbia parviflora L. & Sano dudhe jhar & $\mathrm{H}$ & CL \\
\hline Euphorbiaceae & Euphorbia prostrata Aiton & Sano dudhe jhar & $\mathrm{H}$ & CL \\
\hline Euphorbiaceae & Mallotus philippensis (Lam.) Muell.-Arg. & Sindure & $\mathrm{T}$ & $\mathrm{RF}$ \\
\hline Euphorbiaceae & Phyllanthus emblica L. & Amala & $\mathrm{T}$ & $\mathrm{RF}$ \\
\hline Euphorbiaceae & Phyllanthus glaucas Wall. ex Muell.-Arg. & & $\mathrm{S}$ & GL \\
\hline Euphorbiaceae & Pbyllanthus urinaria L. & Bhuiamala & $\mathrm{H}$ & TF, RF, CL \\
\hline Euphorbiaceae & Phyllanthus virgatus $\mathrm{G}$. Forst. & Amala jhar & $\mathrm{H}$ & CL \\
\hline Euphorbiaceae & Trewia nudiflora L. & Vellar & $\mathrm{T}$ & TF, RF, NF, CL \\
\hline Gentianaceae & Canscora decussata (Roxb.) Schult. \& Schult. f. & Seto phule jhar & $\mathrm{H}$ & $\mathrm{TF}$ \\
\hline Juncaceae & Juncus prismatocarpus R. Br. & Gund & $\mathrm{H}$ & $\mathrm{CL}$ \\
\hline Labiatae & Acrocephalus indicus (Burm. f.) Kuntze & Lerui jhangi (D) & $\mathrm{H}$ & TF, CL \\
\hline
\end{tabular}


Table 2. Cont.

\begin{tabular}{|c|c|c|c|c|}
\hline Family & Specific epithet & Nepali name & Life form & Distribution \\
\hline Labiatae & Anisomeles indica (L.) Kuntze & & $\mathrm{H}$ & TF, NF \\
\hline Labiatae & Colebrookea oppositifolia Sm. & Dhurseli & S & $\mathrm{RF}, \mathrm{NF}$ \\
\hline Labiatae & Hyptis suaveolens (L.) Poit. & Jangali silam & $\mathrm{H}$ & TF, CL \\
\hline Labiatae & Isodon coetsa (Buch.-Ham. Ex D. Don) Kudo & & $\mathrm{H}$ & $\mathrm{NF}$ \\
\hline Labiatae & Isodon ternifolia (D. Don) & Bhimsenpati jhar & S & $\mathrm{RF}$ \\
\hline Labiatae & Leucas cephalotes (Rottb.) Spreng. & Gumpate & $\mathrm{H}$ & CL \\
\hline Labiatae & Lencas indicus (L.) R. Br. Ex Vatke (L. lavandulaefolia Smith) & Gumpate & $\mathrm{H}$ & $\mathrm{RF}$ \\
\hline Labiatae & Leucas mollissima Wall. ex Benth. & Gumpate & $\mathrm{H}$ & $\mathrm{NF}$ \\
\hline Labiatae & Leucas plukenetii (Roth) Spreng. & Gumpate & $\mathrm{H}$ & $\mathrm{CL}$ \\
\hline Labiatae & Mosla dianthera (Buch.-Ham. ex Roxb.) Maxim. & & $\mathrm{H}$ & $\mathrm{RF}$ \\
\hline Labiatae & Ocimum basilicum $\mathrm{L}$. & Babari & $\mathrm{H}$ & TF, NF \\
\hline Labiatae & Pogostemon benghalensis (Burm.f.) Hassk. & Rudilo & $\mathrm{H}$ & RF, NF, CL \\
\hline Labiatae & Salvia plebeia R. Br. & & $\mathrm{H}$ & RF, GL, CL \\
\hline Lauraceae & Litsea chartacea (Wall. ex Nees) Hook. f. & & $\mathrm{T}$ & NF \\
\hline Lauraceae & Litsea monopetala (Roxb.) Pers. & Kutmiro & $\mathrm{T}$ & TF, RF, GL, CL \\
\hline Lecythidaceae & Careya arborea Roxb. & Kumbhi & $\mathrm{T}$ & $\mathrm{TF}, \mathrm{RF}$ \\
\hline \multirow[t]{2}{*}{ Leeaceae } & Leea crispa Royen ex L. & Guithe Padari & & \\
\hline & & Padari & S & $\mathrm{TF}, \mathrm{RF}$ \\
\hline Leeaceae & Leea macrophylla Roxb. ex Hornem. & Galeni & S & $\mathrm{TF}, \mathrm{RF}$ \\
\hline Leguminosae & Acacia catechu (L. f.) Willd. & Khayer & $\mathrm{T}$ & $\mathrm{RF}$ \\
\hline Leguminosae & Acacia pennata (L.) Willd. & Ararikanda & CS & TF, NF \\
\hline Leguminosae & Alysicarpus vaginalis (L.) DC. & & $\mathrm{H}$ & CL \\
\hline Leguminosae & Atylosia scarabaeoides (L.) Benth. & Bangahat & $\mathrm{H}$ & $\mathrm{TF}$ \\
\hline Leguminosae & Baubinia malabarica Roxb. & Amili & $\mathrm{T}$ & $\mathrm{RF}$ \\
\hline Leguminosae & Baubinia vablii Wight \& Arn. & Bhorla & WC & $\mathrm{TF}$ \\
\hline Leguminosae & Butea monosperma (Lam.) Kuntze & Palans & $\mathrm{T}$ & $\mathrm{RF}$ \\
\hline Leguminosae & Cassia fistula $\mathrm{L}$. & Rajbrikchhya & $\mathrm{T}$ & $\mathrm{TF}, \mathrm{RF}$ \\
\hline Leguminosae & Cassia occidentalis $\mathrm{L}$. & Bhaise tapre & $\mathrm{H} / \mathrm{S}$ & TF, CL \\
\hline Leguminosae & Cassia tora $\mathrm{L}$. & Tapre jhar & $\mathrm{H}$ & TF, CL \\
\hline Leguminosae & Codariocalyx motorius (Houttuyn) Ohashi & & S & $\mathrm{TF}, \mathrm{RF}$ \\
\hline Leguminosae & Crotalaria pallida Ait. & & $\mathrm{H}$ & RF, GL \\
\hline \multirow[t]{3}{*}{ Leguminosae } & Crotalaria prostrata Rottb. ex Willd. & Chhinchhine & & \\
\hline & & baja/ Chepte & & \\
\hline & & kuro/ Bilhul & $\mathrm{H}$ & TF, GL, CL \\
\hline Leguminosae & Crotalaria sessiliflora $\mathrm{L}$. & Chhinchhine baja & $\mathrm{H}$ & GL \\
\hline Leguminosae & Crotalaria sp. & Boksi ghanger & $\mathrm{H}$ & TF, RF \\
\hline Leguminosae & Crotalaria sp. & Chhippi & $\mathrm{H}$ & GL \\
\hline Leguminosae & Dalbergia latifolia Roxb. & Satisal & $\mathrm{T}$ & $\mathrm{TF}, \mathrm{RF}$ \\
\hline Leguminosae & Dalbergia sissoo Roxb. ex DC. & Sisau & $\mathrm{T}$ & NF \\
\hline Leguminosae & Desmodium gangeticum (L.) DC. & Bhattamase jhar & S & RF, GL, CL \\
\hline Leguminosae & Desmodium laxiflorum DC. & & S & RF, GL, CL \\
\hline Leguminosae & Desmodium oojeinense (Roxb.) H. Ohashi & Sandan & $\mathrm{T}$ & $\mathrm{TF}$ \\
\hline Leguminosae & Desmodium podocarpon (Poir.) DC. & & $\mathrm{H}$ & $\mathrm{TF}$ \\
\hline Leguminosae & Desmodium triflorum (L.) DC. & Chariamiliki mausi & iH & TF, RF, GL, CL \\
\hline Leguminosae & Desmodium volutinum (Willd.) DC. & & S & $\mathrm{RF}$ \\
\hline \multirow[t]{2}{*}{ Leguminosae } & Flemingia macrophylla (Willd.) Merr. & Bhattamasi & & \\
\hline & & Tinpate ghans & S & TF, RF, GL \\
\hline Leguminosae & Flemingia strobilifera (L.) W. T. Aiton & Bhatmas jhar & S & $\mathrm{TF}, \mathrm{RF}$ \\
\hline Leguminosae & Indigofera linifolia (L. f.) Retz. & & $\mathrm{H}$ & CL \\
\hline Leguminosae & Indigofera pulchella Roxb. & Sagino & $S$ & $\mathrm{TF}, \mathrm{RF}$ \\
\hline
\end{tabular}


Table 2. Cont....

\begin{tabular}{|c|c|c|c|c|}
\hline Family & Specific epithet & Nepali name & Life form & Distribution \\
\hline Leguminosae & Lens culinaris Medikus & Musuro & $\mathrm{H}$ & $\mathrm{CL}$ \\
\hline Leguminosae & Medicago lupulina L. & Jhuppe pyauli & $\mathrm{H}$ & CL \\
\hline Leguminosae & Millettia extensa (Benth.) Baker & Gaujo & WC & TF, RF \\
\hline Leguminosae & Mimosa pudica L. & Lajabati & $\mathrm{H} / \mathrm{S}$ & CL \\
\hline Leguminosae & Phaseolus mungo L. & Ban masyang & $\mathrm{HC}$ & TF, RF \\
\hline Leguminosae & Pbyllodium pulchellum (L.) Desv. & Kanni jhangi & $\mathrm{S}$ & TF, GL \\
\hline Leguminosae & Smithia sensitiva Ait. & Lajaune jhar & $\mathrm{H}$ & TF, RF, CL \\
\hline Leguminosae & Spatholobus parviflorus (Roxb.) Kuntze & Debre lahara, & & \\
\hline \multirow[b]{2}{*}{ Leguminosae } & \multirow[b]{2}{*}{ Tadehagi triquetrum (L.) Ohashi } & Madane, Sal lahara, & WC & TF, RF \\
\hline & & $\begin{array}{l}\text { Jhumke lahara, } \\
\text { Suruwale jhar }\end{array}$ & $\mathrm{S}$ & $\mathrm{TF}$ \\
\hline Leguminosae & Uraria lagapodioides (L.) Desv. & & S & TF, RF, CL \\
\hline Leguminosae & Uraria lagopus DC. & Nilo tanki & S & TF, RF \\
\hline Leguminosae & Vicia angustifolia $\mathrm{L}$. & Kutlikosa & $\mathrm{H}$ & CL \\
\hline Leguminosae & Vicia tetrasperma Moench. & Aankura & $\mathrm{H}$ & TF, CL \\
\hline Liliaceae & Asparagus racemosus Willd. & Kurilo & $\mathrm{H}$ & $\mathrm{RF}$ \\
\hline Liliaceae & Chlorophytum nepalense (Lindl.) Baker & Pyaje & $\mathrm{H}$ & $\mathrm{TF}$ \\
\hline Liliaceae & Smilax ovalifolia Roxb. ex D. Don & Kukur daino & CS & TF, RF \\
\hline Lythraceae & Ammannia baccifera $\mathrm{L}$. & & $\mathrm{H}$ & $\mathrm{RF}$ \\
\hline Lythraceae & Lagerstroemia parviflora Roxb. & Botdhairo & $\mathrm{T}$ & $\mathrm{TF}, \mathrm{RF}$ \\
\hline Lythraceae & Rotala indica (Willd.) Koehne & Belauti jhar & $\mathrm{H}$ & TF, CL \\
\hline Lythraceae & Rotala rotundifolia (Buch.-Ham. ex Roxb.) Koehne & Ambapate jhar & $\mathrm{H}$ & CL \\
\hline Malvaceae & Kydia calycina Roxb. & Kubindo & S & $\mathrm{TF}, \mathrm{RF}$ \\
\hline Malvaceae & Sida rhombifolia L. & Balu & $\mathrm{H} / \mathrm{S}$ & TF, RF, NF, CL \\
\hline Malvaceae & Thespesia lampas (Cav.) Dalz. et Gibs. & Ban kapas & S & $\mathrm{TF}, \mathrm{NF}$ \\
\hline \multirow[t]{2}{*}{ Malvaceae } & \multirow[t]{2}{*}{ Urena lobata L. } & Balu /Chhipi, & & \\
\hline & & Bhendichirchut & $\mathrm{H}$ & TF, NF, CL \\
\hline Marsileaceae & Marsilea crenata Presl & Charpate jhar & $\mathrm{H}$ & CL \\
\hline Melastomataceae & Osbeckia stellata Buch.-Ham. ex D. Don & Angeri & $\mathrm{S}$ & $\mathrm{TF}$ \\
\hline Meliaceae & Azadirachta indica A. Juss. & Nim & $\mathrm{T}$ & NF, CL \\
\hline Meliaceae & Melia azedarach L. & Bakaino & $\mathrm{T}$ & $\mathrm{NF}, \mathrm{CL}$ \\
\hline Menispermaceae & Cissampelos pareira L. & Batulpate & $\mathrm{HC}$ & TF, RF, NF \\
\hline Menispermaceae & Stephania elegans Hook. f. \& Thomson & Batulpate & $\mathrm{HC}$ & $\mathrm{RF}, \mathrm{NF}, \mathrm{CL}$ \\
\hline Menispermaceae & Stephania japonica var. discolor (Miq.) Forman & Batulpate & $\mathrm{HC}$ & TF, GL \\
\hline Menispermaceae & Tinospora sinensis (Lour.) Merr. & Gudargano & $\mathrm{HC}$ & NF \\
\hline Myrsinaceae & Maesa chisia Buch.-Ham. ex D. Don & Bilaune & S & TF, RF \\
\hline \multirow[t]{2}{*}{ Myrsinaceae } & \multirow[t]{2}{*}{ Myrsine semiserrata Wall. } & Kalikath & & \\
\hline & & (Karauta) & $\mathrm{S} / \mathrm{T}$ & TF, NF \\
\hline Myrsinaceae & Myrsine sp. & Damai kath & $\mathrm{T}$ & TF, RF, NF \\
\hline Myrtaceae & Cleistocalyx operculatus (Roxb.) Merr. \& Perry & Kyamun & $\mathrm{T}$ & TF, RF, GL \\
\hline Myrtaceae & Syzygium cumini (L.) Skeels & Jamun & $\mathrm{T}$ & TF, RF, CL \\
\hline Onagraceae & Ludwigia perennis L. & Lwang jhar & $\mathrm{H}$ & TF, CL \\
\hline Ophioglossaceae & Ophioglosum petiolatum Hook. & Jibre sag & $\mathrm{H}$ & TF, RF, NF, \\
\hline Orchidaceae & Eulophia berbacea Lindl. & & $\mathrm{H}$ & CL \\
\hline Orchidaceae & Zeuxine flava (Lindl.) Trimen & & $\mathrm{H}$ & $\mathrm{NF}, \mathrm{CL}$ \\
\hline Orchidaceae & Zeuxine strateumatica (L.) Schlechter & & $\mathrm{H}$ & CL \\
\hline Oxalidaceae & Oxalis corniculata $\mathrm{L}$. & Chariamili & $\mathrm{H}$ & TF, RF, NF, CL \\
\hline Piperaceae & Piper longum L. & Pipla & $\mathrm{HC}$ & TF, RF, NF, CL \\
\hline Poaceae & Apluda mutica $\mathrm{L}$. & & $\mathrm{H}$ & $\mathrm{RF}, \mathrm{NF}$ \\
\hline Poaceae & Arundinella benghalensis (Spreng.) Druce & & $\mathrm{H}$ & CL \\
\hline
\end{tabular}


Table 2. Cont

\begin{tabular}{|c|c|c|c|c|}
\hline Family & Specific epithet & Nepali name & Life form & Distribution \\
\hline Poaceae & Axonopus compressus (Swartz) P. Beauv. & $\begin{array}{l}\text { Makai banso/ } \\
\text { Thulo banso }\end{array}$ & $\mathrm{H}$ & TF, CL \\
\hline Poaceae & Bothriochloa glabra (Roxb.) A. Camus & & $\mathrm{H}$ & $\mathrm{TF}, \mathrm{RF}$ \\
\hline Poaceae & Bothriochloa intermedia (R. Br.) A. Camus & & $\mathrm{H}$ & $\mathrm{TF}$ \\
\hline Poaceae & Brachiaria distachya (L.) Stapf & Banso & $\mathrm{H}$ & TF, CL \\
\hline Poaceae & Brachiaria miliiformis (Presl \& C. Presl) A. Chase & Banso & $\mathrm{H}$ & $\mathrm{TF}$ \\
\hline Poaceae & Brachiaria mutica (Forssk.) Stapf & Banso & $\mathrm{H}$ & CL \\
\hline Poaceae & Brachiaria spp. & Banso & $\mathrm{H}$ & TF, RF, NF, CL \\
\hline Poaceae & Chloris dolichostachya Lagasca & & $\mathrm{H}$ & $\mathrm{RF}$ \\
\hline Poaceae & Chrysopogon aciculatus (Retz.) Trin. & Kuro ghans & $\mathrm{H}$ & TF, NF, CL \\
\hline Poaceae & Cymbopogon jwarancusa (Jones) Schult. & & $\mathrm{H}$ & CL \\
\hline Poaceae & Cymbopogon osmastonii R. N. Parker & & $\mathrm{H}$ & GL \\
\hline Poaceae & Cynodon dactylon (L.) Pers. & Dubo & $\mathrm{H}$ & TF, RF, NF, GL, CL \\
\hline Poaceae & Desmostachys bipinnata (L.) Stapf & Kush & $\mathrm{H}$ & TF, RF, GL, CL \\
\hline Poaceae & Dicanthium annulatum (Forssk.) Stapf & Jhuse ankhle jhar & $\mathrm{H}$ & CL \\
\hline Poaceae & Digitaria ciliaris (Retz.) Koeler & Chitre banso & $\mathrm{H}$ & TF, NF \\
\hline Poaceae & Digitaria longiflora (Retz.) Pers. & Chitre banso & $\mathrm{H}$ & CL \\
\hline Poaceae & Digitaria spp. & Chitre banso & $\mathrm{H}$ & CL \\
\hline Poaceae & Echinochloa colona (L.) Link & Sama ghans & $\mathrm{H}$ & TF, CL \\
\hline Poaceae & Eleusine indica (L.) Gaertn. & Kode jhar & $\mathrm{H}$ & TF, CL \\
\hline Poaceae & Eragrostis atrovirens (Desf.) Trin ex Steudel & Banso & $\mathrm{H}$ & CL \\
\hline Poaceae & Eragrostis tenella (L.) Beauvois ex Roem. \& Schultes & Junge banso & $\mathrm{H}$ & TF, NF, GL, CL \\
\hline Poaceae & Eragrostris unioloides (Retz.) Nees ex Steudel & Chiure banso & $\mathrm{H}$ & $\mathrm{TF}, \mathrm{NF}, \mathrm{CL}$ \\
\hline Poaceae & Hemarthria compressa (L. f.) R. Br. & Ghode dubo & $\mathrm{H}$ & TF, RF, GL, CL \\
\hline Poaceae & Imperata cylindrica (L.) P. Beauv. & Siru & $\mathrm{H}$ & TF, RF, NF, GL, CL \\
\hline Poaceae & Isachne globosa (Thunb.) Kuntze & & $\mathrm{H}$ & CL \\
\hline Poaceae & Ischaemum rugosum Salisb. & Madilo & $\mathrm{H}$ & CL \\
\hline Poaceae & Leersia hexandra Swartz & Karaute ghans & $\mathrm{H}$ & CL \\
\hline Poaceae & Oplismenus burmannii (Retz.) P. Beauv. & Ote banso & $\mathrm{H}$ & TF, RF, CL \\
\hline Poaceae & Oplismenus compositus (L.) P. Beauv. & Ote banso & $\mathrm{H}$ & $\mathrm{TF}, \mathrm{NF}$ \\
\hline Poaceae & Panicum notatum Retz. & & $\mathrm{H}$ & $\mathrm{RF}$ \\
\hline Poaceae & Paspalum distichum L. & Mane banso & $\mathrm{H}$ & $\mathrm{TF}$ \\
\hline Poaceae & Paspalum scrobiculatum $\mathrm{L}$. & Mane banso & $\mathrm{H}$ & TF, RF, NF, CL \\
\hline Poaceae & Phragmites karka (Retz.) Trin. ex Steudel & Masino narkat & $\mathrm{H}$ & RF, GL, CL \\
\hline Poaceae & Pogonatherum crinitum (Thunb.) Kunth & Khari banso & $\mathrm{H}$ & TF, CL \\
\hline Poaceae & Polypogon monspeliensis (L.) Desf. & Puchhre jhar & $\mathrm{H}$ & CL \\
\hline Poaceae & Pseudopogonatherum contortum (Brongn.) A. Camus & & $\mathrm{H}$ & TF, RF \\
\hline Poaceae & Saccharum benghalense Retz. & Worela & $\mathrm{H}$ & RF, GL \\
\hline Poaceae & Saccharum spontaneum $\mathrm{L}$. & Kans; Th: Jhaksi & $\mathrm{H}$ & TF, RF, NF, GL, CL \\
\hline Poaceae & Sacciolepis indica (L.) A. Chase & & $\mathrm{H}$ & TF, CL \\
\hline Poaceae & Sacciolepis myosuroides (R. Br.) A. Camus & & $\mathrm{H}$ & $\mathrm{TF}$ \\
\hline Poaceae & Setaria pallide-fusca (Schumach.) Stapf \& C. E. Hubbard & $\begin{array}{l}\text { Bale banso/ } \\
\text { Jhusile banso }\end{array}$ & $\mathrm{H}$ & TF, CL \\
\hline Poaceae & Sporobolus diander (Retz.) P. Beauv. & & $\mathrm{H}$ & CL \\
\hline Poaceae & Themeda arundinacea (Roxb.) Ridley & Khadahi & $\mathrm{H}$ & TF, RF, GL \\
\hline Polygalaceae & Polygala sp. & & $\mathrm{H}$ & CL \\
\hline Polygonaceae & Polygonum barbatum L. & Pire bikh & $\mathrm{H}$ & $\mathrm{NF}, \mathrm{CL}$ \\
\hline Polygonaceae & Polygonum bydropiper L. & Pire jhar & $\mathrm{H}$ & $\mathrm{TF}$ \\
\hline Polygonaceae & Polygonum lanigerum $\mathrm{R}$. Br. & Pire bish & $\mathrm{H}$ & $\mathrm{CL}$ \\
\hline Polygonaceae & Polygonum plebeium R. Br. & Sukul jhar & $\mathrm{H}$ & TF, RF, CL \\
\hline
\end{tabular}


Table 2. Cont....

\begin{tabular}{|c|c|c|c|c|}
\hline Family & Specific epithet & Nepali name & Life form & Distribution \\
\hline Pontederiaceae & Monochoria hastata (L.) Solms & Jaluki & $\mathrm{H}$ & $\mathrm{CL}$ \\
\hline Primulaceae & Anagallis arvensis $\mathrm{L}$. & Krishnanil & $\mathrm{H}$ & CL \\
\hline Primulaceae & Androsace umbellata (Lour.) Merr. & Chhate primula & $\mathrm{H}$ & $\mathrm{NF}, \mathrm{CL}$ \\
\hline Pteridaceae & Adiantum caudatum $\mathrm{L}$. & Rani sinka & $\mathrm{H}$ & $\mathrm{RF}$ \\
\hline Pteridaceae & Adiantum lunalatum Buru & Rani sinka & $\mathrm{H}$ & $\mathrm{RF}$ \\
\hline Pteridaceae & Pteris biaurita L. & & $\mathrm{H}$ & $\mathrm{RF}, \mathrm{CL}$ \\
\hline Pteridaceae & Pteris incisa Thunb. & & $\mathrm{H}$ & NF \\
\hline Rhamnaceae & Zizyphus mauritiana Lam. & Bayer & $\mathrm{T}$ & GL \\
\hline Rhamnaceae & Zizyphus nummularia (Burm. f.) Wight \& Arn. & Jangali bayer & S & $\mathrm{TF}, \mathrm{RF}$ \\
\hline Rhamnaceae & Zizyphus rugosa Lam. & Dumarai & $\mathrm{T}$ & $\mathrm{TF}$ \\
\hline Rosaceae & Fragaria indica Andr. & Bhuin kafal & $\mathrm{H}$ & $\mathrm{NF}$ \\
\hline \multirow[t]{2}{*}{ Rosaceae } & Potentilla supina $\mathrm{L}$. & Jangali bajradanti, & & \\
\hline & & Dhaniya pate & $\mathrm{H}$ & RF, CL \\
\hline Rubiaceae & Anthocephalus chinensis (Lam.) A. Rich. ex Walp. & Karam & $\mathrm{T}$ & $\mathrm{RF}$ \\
\hline Rubiaceae & Borreria alata (Aubl.) DC. & & $\mathrm{H}$ & CL \\
\hline Rubiaceae & Borreria articularis (L.f.) F. N. Williams & & $\mathrm{H}$ & TF, RF, CL \\
\hline Rubiaceae & Borreria pusilla (Wall.) DC. & & $\mathrm{H}$ & TF, CL \\
\hline Rubiaceae & Borreria repens DC. & & $\mathrm{H}$ & CL \\
\hline Rubiaceae & Coffea benghalensis Heyne ex Roem et Schult & & S & NF \\
\hline Rubiaceae & Hedyotis brachypoda (A. P. de Candolle) V. P. Sivarajan & & $\mathrm{H}$ & $\mathrm{TF}$ \\
\hline Rubiaceae & Hedyotis corymbosa (L.) Lam. & & $\mathrm{H}$ & TF, CL \\
\hline Rubiaceae & Hedyotis diffusa Willd. & & $\mathrm{H}$ & TF, CL \\
\hline Rubiaceae & Hedyotis gracilis Hook. f. & & $\mathrm{H}$ & CL \\
\hline Rubiaceae & Hedyotis lineata Roxb. & & $\mathrm{H}$ & $\mathrm{TF}$ \\
\hline Rubiaceae & Mitragyna parviflora (Roxb.) Korth. & Kaim & $\mathrm{T}$ & $\mathrm{RF}$ \\
\hline Rubiaceae & Wendlandia puberula DC. & Kainyo & $\mathrm{T}$ & $\mathrm{TF}$ \\
\hline Rubiaceae & Xeromphis spinosa (Thunb.) Keay & Mainkanda & $\mathrm{S}$ & TF, RF, NF, GL \\
\hline Rubiaceae & Xeromphis uliginosa (Retz.) Maheshwari & Pidar & $\mathrm{T}$ & $\mathrm{RF}$ \\
\hline Rutaceae & Clausena pentaphylla DC. & & S & $\mathrm{TF}, \mathrm{RF}$ \\
\hline Rutaceae & Murraya koenigii (L.) Spreng. & Asare & $\mathrm{S}$ & TF, RF, NF, GL \\
\hline Rutaceae & Skimmia arborescens T. Anderson ex Gamble & Asare & $\mathrm{S}$ & TF, RF \\
\hline Sapindaceae & Schleichera oleosa (Lour.) Oken & Kusum & $\mathrm{T}$ & $\mathrm{TF}, \mathrm{RF}$ \\
\hline Schizaeaceae & Lygodium flexuosum (L.) Sw. & Janai lahara & $\mathrm{HC}$ & TF, RF \\
\hline Scrophulariaceae & Bacopa hamiltoniana (Benth.) Wettst. & & $\mathrm{H}$ & $\mathrm{RF}$ \\
\hline Scrophulariaceae & Bacopa monnieri (L.) Pennell & & $\mathrm{H}$ & $\mathrm{TF}$ \\
\hline Scrophulariaceae & Lindernia anagallis (Burm. f.) Pennell & & $\mathrm{H}$ & CL \\
\hline Scrophulariaceae & Lindernia antipoda (L.) Alston & & $\mathrm{H}$ & CL \\
\hline Scrophulariaceae & Lindernia ciliata (Colsm.) Pennell & Karaute jhar & $\mathrm{H}$ & $\mathrm{CL}$ \\
\hline Scrophulariaceae & Lindernia crustacea (L.) F. Muell. & & $\mathrm{H}$ & TF, CL \\
\hline Scrophulariaceae & Lindernia oppositifolia (L.) Mukerjee & & $\mathrm{H}$ & CL \\
\hline Scrophulariaceae & Lindernia parviflora (Roxb.) Haines & & $\mathrm{H}$ & CL \\
\hline Scrophulariaceae & Lindernia pusilla (Willd.) Bold. & & $\mathrm{H}$ & CL \\
\hline Scrophulariaceae & Lindernia sp. & & $\mathrm{H}$ & GL \\
\hline Scrophulariaceae & Mazus pumilus (Burm. f.) Steenis & & $\mathrm{H}$ & CL \\
\hline Scrophulariaceae & Mecardonia procumbens (Mill.) Small & & $\mathrm{H}$ & RF, GL, CL \\
\hline Scrophulariaceae & Scoparia dulcis L. & Chini jhar & $\mathrm{H}$ & CL \\
\hline Scrophulariaceae & Striga gesnerioides (Willd.) Vatke & & $\mathrm{H}$ & CL \\
\hline Scrophulariaceae & Veronica anagallis-aquatica L. & Dhape jhar & $\mathrm{H}$ & CL \\
\hline Selaginellaceae & Selaginella ciliaris (Retz.) Spring & & $\mathrm{H}$ & TF, RF \\
\hline Solanaceae & Physalis divaricata D. Don & Patpate & $\mathrm{H}$ & NF, CL \\
\hline
\end{tabular}


Table 2. Cont.

\begin{tabular}{|c|c|c|c|c|}
\hline Family & Specific epithet & Nepali name & Life form & Distribution \\
\hline Solanaceae & Solanum aculeatissimum Jacq. & Kantakari & $\mathrm{H}$ & NF \\
\hline Solanaceae & Solanum torvum $\mathrm{Sw}$ & Thulo bihid & $\mathrm{H}$ & $\mathrm{NF}$ \\
\hline Solanaceae & Solanum Virginianum L. & Kantakari & $\mathrm{H}$ & RF, NF. GL, CL \\
\hline Sterculiaceae & Helicteres isora $\mathrm{L}$. & Simthi & $\mathrm{S}$ & $\mathrm{TF}, \mathrm{RF}$ \\
\hline Sterculiaceae & Melocbia corchorifolia L. & Bankuro & $\mathrm{H}$ & CL \\
\hline Sterculiaceae & Pterospermum acerifolium (L.) Willd. & Singane & $\mathrm{T}$ & $\mathrm{RF}$ \\
\hline Tamaricaceae & Tamarix dioica Roxb. ex Roth. & Jheuwa & $\mathrm{S} / \mathrm{T}$ & RF, CL \\
\hline Theaceae & Schima wallichii (DC.) Korth & Chilaune & $\mathrm{T}$ & $\mathrm{TF}$ \\
\hline Thelypteridaceae & Thelypteris auriculata (J. Sm.) K. Iwats. & Bishkoche & $\mathrm{H}$ & TF, RF, NF, GL, CL \\
\hline Tiliaceae & Corchorus aestuans $\mathrm{L}$. & Ban pat & $\mathrm{H}$ & TF, CL \\
\hline Tiliaceae & Grewia disperma Rottb. & & $S$ & $\mathrm{RF}$ \\
\hline Tiliaceae & Grewria belicterifolia Wall. ex G. Don & Kharbuja & $\mathrm{H}$ & TF, RF \\
\hline Tiliaceae & Grewia sapida Roxb. Ex DC. & Pharsa & S & TF, RF, GL \\
\hline Tiliaceae & Grewia subinaequalis DC. & Dafer & $\mathrm{T}$ & $\mathrm{TF}, \mathrm{RF}$ \\
\hline Tiliaceae & Triumfetta rhomboides Jacq. & Dalle kuro & $\mathrm{H}$ & CL \\
\hline Typhaceae & Typha angustifolia $\mathrm{L}$. & Pater & $\mathrm{H}$ & RF, GL, CL \\
\hline Urticaceae & Gonostegia pentandra (Roxb.) Miq. & Sim chiple jhar & $\mathrm{H}$ & GL, CL \\
\hline Urticaceae & Pouzolzia zeylanica (L.) J. Bennett \& R. Br. & Chiple jhar & $\mathrm{H}$ & $\mathrm{RF}, \mathrm{CL}$ \\
\hline Verbenaceae & Callicarpa macrophylla Vahl & Dhaichamla & $\mathrm{S}$ & $\mathrm{RF}$ \\
\hline Verbenaceae & Clerodendrum viscosum Vent. & Bhanthi & S & TF, RF, NF, CL \\
\hline Verbenaceae & Gmelina arborea Roxb. & Khamari & $\mathrm{T}$ & $\mathrm{RF}$ \\
\hline Verbenaceae & Pbyla nodiflora (L.) Greene & Bhuin okra & $\mathrm{H}$ & TF, RF, GL, CL \\
\hline Verbenaceae & Premna integrifolia $\mathrm{L}$. & Gindari & $\mathrm{T}$ & TF, NF \\
\hline Verbenaceae & Vitex negundo L. & Simali & S & $\mathrm{CL}$ \\
\hline Vitaceae & Cissus repens Lam. & Charchare lahara & $\mathrm{S}$ & TF, NF \\
\hline Vitaceae & Tetrastigma dubium (M. A. Lawson) Planch. & & $\mathrm{HC}$ & $\mathrm{RF}$ \\
\hline Zingiberaceae & Alpinia sp. & & $\mathrm{H}$ & RF \\
\hline Zingiberaceae & Costus speciosus (J. Koenig) Smith & Bet lauri & $\mathrm{H}$ & $\mathrm{TF}$ \\
\hline
\end{tabular}

$\mathrm{TF}=$ Tikauli forest, $\mathrm{RF}=$ Royal Chitwan National Park Forest, $\mathrm{NF}=$ Forests along Narayani river, $\mathrm{GL}=$ Grasslands of National Park Forest, and $\mathrm{CL}=$ common landa

The largest genera are listed in Table 3. Lindernia and Desmodium are the two largest genera, with 8 and 6 species, respectively. Crotalaria, Cyperus and Hedyotis represent 3 species each. One hundred ninety-four genera had only one species each. The largest genera of the present study such as Lindernia and Cyperus were also reported among the top 5 genera in the aquatic flora of the plains of eastern Nepal (Siwakoti and Varma, 1998).

Table 3. Genera with 3 to more number of species in the vascular flora of western Chitwan, Nepal

\begin{tabular}{|lc|}
\hline Genera & Total species \\
\hline Lindernia All., Scrophulariaceae & 8 \\
Desmodium Desv., Leguminosae & 6 \\
Crotalaria L., Leguminosae & 5 \\
Cyperus L., Cyperaceae & 5 \\
Hedyotis L., Rubiaceae & 5 \\
Borreria G. Meyer, Rubiaceae & 4 \\
Bracbiaria Griseb., Poaceae & 4 \\
Commelina L., Commelinaceae & 4 \\
Grewia L., Tiliaceae & 4 \\
Leucas R. Br., Labiatae & 4 \\
Phyllanthus L., Euphorbiaceae & 4 \\
Polygonum L., Polygonaceae & 4 \\
Blumea DC., Asteraceae & 3 \\
Cassia L., Leguminosae & 3 \\
Digitaria Heister ex Fabr., Poaceae & 3 \\
Dioscorea L., Dioscoreaceae & 3 \\
Eragrostis Wolf, Poaceae & 3 \\
Euphorbia L., Euphorbiaceae & 3 \\
\hline
\end{tabular}


Table 3. Cont..............

Gnaphalium L., Asteraceae

Solanum L., Solanaceae

Terminalia L., Combretaceae

Zizyphus Mill., Rhamnaceae

\section{Species distribution}

The composition of species varies with the land types of the study plots. Some species are shared between two or more plots and others are unique (Table 4). The highest unique species are found in the common lands (87 spp.), followed by the Royal Chitwan National Park forest (36 spp.) and the Tikauli forest (32 spp.). The Tikauli forest and The National Park Forest (Table 4) shared 44 species (12.607\%) followed by the Tikauli forest and the common land forest (24 species). Ageratum houstonianum Mill., Cynodon dactylon (L.) Pers., Imperata cylindrica (L.) P. Beauv., Rungia parviflora (Retz.) Nees, Saccharum spontaneum L. and Thelypteris auriculata (J. Sm.) K. Iwats are common species distributed in all the five blocks (Table 2, 4). Above species were also reported as common species in Ramput condition (Dangol, 1989-1999).

Table 4. Number of species unique or common to different research blocks in western Chitwan, Nepal

\begin{tabular}{|c|c|c|c|c|c|c|c|c|c|}
\hline \multicolumn{2}{|c|}{ Single block } & \multicolumn{2}{|c|}{ Two blocks } & \multicolumn{2}{|c|}{ Three blocks } & \multicolumn{2}{|c|}{ Four blocks } & \multicolumn{2}{|c|}{ Five blocks } \\
\hline Sites & No. & Sites & No. & Sites & No. & Sites & No. & Sites & No. \\
\hline CL & 87 & $\mathrm{GL}+\mathrm{CL}$ & 2 & $\mathrm{RF}+\mathrm{GL}+\mathrm{CL}$ & 6 & $\mathrm{RF}+\mathrm{NF}+\mathrm{GL}+\mathrm{CL}$ & 3 & $\mathrm{TF}+\mathrm{RF}+\mathrm{NF}+\mathrm{GL}+\mathrm{CL}$ & 6 \\
\hline GL & 8 & $\mathrm{NF}+\mathrm{CL}$ & 10 & $\mathrm{RF}+\mathrm{NF}+\mathrm{CL}$ & 3 & $\mathrm{TF}+\mathrm{NF}+\mathrm{GL}+\mathrm{CL}$ & 2 & & \\
\hline NF & 19 & $\mathrm{RF}+\mathrm{CL}$ & 7 & $\mathrm{TF}+\mathrm{GL}+\mathrm{CL}$ & 1 & $\mathrm{TF}+\mathrm{RF}+\mathrm{GL}+\mathrm{CL}$ & 8 & & \\
\hline $\mathrm{RF}$ & 36 & $\mathrm{RF}+\mathrm{GL}$ & 2 & $\mathrm{TF}+\mathrm{NF}+\mathrm{CL}$ & 4 & $\mathrm{TF}+\mathrm{RF}+\mathrm{NF}+\mathrm{CL}$ & 10 & & \\
\hline \multirow[t]{5}{*}{$\mathrm{TF}$} & 32 & $\mathrm{RF}+\mathrm{NF}$ & 2 & $\mathrm{TF}+\mathrm{RF}+\mathrm{CL}$ & 10 & $\mathrm{TF}+\mathrm{RF}+\mathrm{NF}+\mathrm{GL}$ & 2 & & \\
\hline & & $\mathrm{TF}+\mathrm{CL}$ & 24 & $\mathrm{TF}+\mathrm{RF}+\mathrm{GL}$ & 4 & & & & \\
\hline & & $\mathrm{TF}+\mathrm{GL}$ & 2 & $\mathrm{TF}+\mathrm{RF}+\mathrm{NF}$ & 4 & & & & \\
\hline & & $\mathrm{TF}+\mathrm{NF}$ & 11 & & & & & & \\
\hline & & $\mathrm{TF}+\mathrm{RF}$ & 44 & & & & & & \\
\hline Total & 182 & & 104 & & 32 & & 25 & & 6 \\
\hline$\%$ & 52.15 & & 29.79 & & 9.17 & & 7.16 & & 1.72 \\
\hline
\end{tabular}

$\mathrm{CL}=$ Common lands, $\mathrm{GL}=$ Grasslands (national park); $\mathrm{NF}=$ Narayani river forest; $\mathrm{RF}=$ National park forest; and $\mathrm{TF}=\mathrm{Tikauli}(\mathrm{Barandabar})$ forest

\section{Life forms}

In the study areas, the most prominent life form is herbs (239 spp.) followed by trees (46 spp.), shrubs (35 spp.), climbing herbs (15 spp.), and climbing shrub (4 spp.) (Table 2). The dominance of herns was also reported by Jha and Jha (2000) in the flora of Morang district and adjoining areas of Nepal. Herbs are the main component of the common lands, grasslands and ground flora of the forest vegetation. The herbaceous vegetation in the grasslands are represented by tall grasses like Themeda arundinacea (Roxb.) Ridley, Saccharum benghalense Retz., Saccharum spontaneum L. and Imperata cylindrica (L.) P. Beauv., Spatholobus parviflorus (Roxb.) Kuntze, Millettia extensa (Benth.) Baker and Baubinia vablii Wight \& Arn. are three woody climbers of the forests of the western Chitwan. Merremia vitifolia (Burm. f.) Hallier f. is a herbaceous trailer that was recorded in the Tikauli forest.

\section{Folk nomenclature of plants}

Of the listed plants, many plants have local names either in Nepalese or other tribal languages (Table 2). The plants with less economic value generally lack local names. For instance, the small plants like Androsace umbellate (Lour.) Merr., Canscorea decussata (Roxb.) Schult. \& R.S. Schult. f., Hemigraphis hirta (Vahl) T. Anderson, etc. do not have vernacular names. Plants are named in different ways on the basis of habit, habitat, smell, test, and morphological characters, which are also the basis of nomenclature in plant taxonomy.

The local names are given on the basis of structure, color, smell, etc. The Nepalese call "Tinpate" for the plants that possess three leaves and refer to the generas such as Flemingia, Uraria, and Desmodium of the Leguminosae family. Similarly, "Gandhe" meaning a bad or unpleasant smell, stands for the plants (Hyptis suaveolens (L.) Poit., Ageratum conyzoides L., Ageratum houstonianum Merr.). In Nepali, "Chariamili” stands for three species: Oxalis corniculata L., O. corymbosa DC. and O. latifolia Humb. The local name "Boki jhar", meaning goat weed, is 
given to Gnaphalium purpureum L., Gnaphalium polycaulon Pers. and Gnaphalium affine D. Don distributed in tropical and subtropical areas of Nepal. The "lahara" is given to the climbering plants. For instance, the woody climber, Spatholobus parviflorus (Roxb.) Kuntze, known by "Debre lahara", has the habit or characteristic of climbing up to the left. "Debre" means left in Nepali. "Sal lahara" is the name given to the woody climber Spatholobus parviflorus (Roxb.) Kuntze, plant that climbs on "Sal" (Shorea robusta Gaertn.). Some of the authors searched the m eaning of local names for the plants and found simiar naming systems based on smell, habit, habitats, color of flower (Dangol, 2002-2003; Pohle, 1990; Sacherer, 1997). The local names for plants may create confusion when one local name stands for more than two types (species) of plants or when one plant has more than one local name. In such cases, those who need the botanical name should consult experts or authentic herbarium specimens for proper identifiction.

\section{CONCLUSIONS}

This species list includes only those plant species, which were encountered in or near the research plots in western Chitwan. These plot locations were designed to represent the diversity of land types in the region. For the taxonomic purposes, it is necessary to collect plant specimens from different locations of the entire district. These collections will allow us to recognize plant species correctly and follow changes in flora diversity and study how they are related to population change. Comprehensive interdisciplinary studies on plants and population are recommended to explore the reciprocal relations between population and the environment. Collaborations such as these between botanists and social scientists will help us better understand the connection between plants, people and culture.

\section{ACKNOWLEDGEMENTS}

This research was supported by a grant from the National Institute of Child Health and Human Development (Grant \# ROl-HD 33551), USA. Sincere thanks are extended to W. G. Axinn (Principal Investigator), G. P. Shivakoti, Jennifer Barber, Kerry Richter, Dirgha Ghimire, Prem Bhandari and Kishor Gajurel for advice during the sample design stages and Lisa D. Pearce for editing the previous version of the manuscript. Netra Chhetri, Sujan Shrestha, Harimaya Poudel, Rajendra Darai, Janaki Rimal, Binoj Shrestha, Shree Bhakta Dhungana and the rest of the PERL Staff are also thanked for their assistance. Thanks are also extended to the Department of National Parks and Wildlife Conservation and Department of Forest, Kathmandu, Nepal for permission of conducting this research in the forests.

\section{REFERENCES CITED}

Bhuju, D. R. and P. Rana. 2000. An appraisal of human impact on vegetation in high altitudes (Khumbu region) of Nepal. Nep. J. Sci. Tech. 2:101-105.

Cosgriff, K., N. Lipscombe and J. Bauer. 1999. Tourist/wildlife interactions in Royal Chitwan National Park, Nepal. Ecoprint 6(1):1-7.

Dangol, D. R. 1998-1999. An inventory of biodiversity of Rampur, Chitwan, Nepal. J. Inst. Agric. Anim. Sci. 18-19:27-40.

Dangol, D. R. and 2002-2003. Folk plant nomenclature of Tharu tribe of Chitwan, Central Nepal. NAHSON Bull. 12-13:2-4.

Dangol, D. R. and S. B. Gurung, 1988. Preliminary survey of major field crops weeds and farmers weed management practices in Chitwan, Nepal. A report submitted to RONAST, Kathmandu, Nepal.

Dangol, D. R. and S. B. Gurung, 1989. Floristic composition and dominance of weeds in summer maize fields in the Chitwan valley of Nepal. J. Inst. Agric. Anim. Sci. 10:57-66.

Dinerstein, E, 1992. Effects of Rhinoceros unicornis on riverine forest structure in lowland Nepal. Ecology 73(2):701-704.

Ghimire, S. K., J. P. Sah, K. K. Shrestha and D. Bajracharya. 1999. Ecological study of some high altitude medicinal and aromatic plants in the Gyasumdo valley, Manang, Nepal. Ecoprint 6(1):17-25.

Hara, H, and L. H. J. Willians (eds.), 1979. An enumeration of the flowering plants of Nepal. Vol. 2 British Museum (Natural History) Publication. London.

Hara, H., A. O. Chater and L. H. J. Williams (eds.) 1982. An enumeration of the flowering plants of Nepal. Vol. 3. British Museum (Natural History) Publication London. 
IUCN. 1994. Traditional uses of forests: report on the national survey. IUCN-The World Conservation Union, IUCN-Sri Lanka, The Social Policy Service, Ministry of Lands, Irrigation and Mahaweli Development, Forest Department, Sri Lanka.

Jha, S. and P. K. Jha. 2000. Contributions to the flora of Morang district and adjoining areas of Nepal. A Norwegian J. Bot. 5(1-2) : 25-64.

Mabberley, D. J. 1987. The plant-book: a portable dictionary of the higher plants. Cambridge University Press, NY, USA.

Mathews, S., G. Shivakoti and Chhetri N. 2000. Population forces and environmental change: observations from western Chitwan, Nepal. Soc. Nat. Resour. 13: 763-775.

Penitsa, M., P. Dimopoulos, G. Iatrou and D. Tzanoudakis. 1994. Contributions to the study of the Greek flora: flora and vegetation of the Enousses (Oinousses) islands (E. Aegean area). Flora 189:69-78.

Pohle, P. 1990. Useful plants of Manag district: A contribution to the ethnobotany of the Nepal-Himalaya. Franz Steiner Verlag Woesbaded GMBH. Stuttgart.

Press, J. R., K. K. Shrestha and D. A. Sutton. 2000. Annotated checklist of the flowering plants of Nepal. The Natural History Museum, London.

Quattrocchi, U. 2000. CRC world dictionary of plant names: common names, scientific names, aponyms, synonyms, and etymology. Vol. I-IV. CRC Press, USA.

Rajbhandary, T. K., M. S. Bista and V. L Gurung. (eds.). 1994. Enumeration of the vascular plants of west Nepal. HMG/N, Ministry of Forests and Soil Conservation, Department of Plant Resources, Thapathali, Kathmandu, Nepal.

Roy, S. and R. K. Pokharel. 2000. Impact of community forest on rural livelihood. Banko Janakari 10(2):54-56.

Sacherer, J. 1979. The high altitude ethnobotany of the Rolwaling Sherpas. Contributions to Nepalese Studies. 6(2):45-64.

Siwakoti, M. and S. K. Varma. 1998. Aquatic flora of the plains (Biratnagar-Dharan) of Eastern Nepal. Higher Plants of Indian Sub-continent 8:53-67.

Turner I. M. 1997. A tropical flora summarized - a statistical analysis of the vascular plant diversity of Malay. Flora 192:157-163. 